\title{
Conformational differences among metarhodopsin I, metarhodopsin II, and opsin probed by wide-angle $X$ - ray scattering
}

\section{$\operatorname{AUTHOR}(\mathrm{S})$ :}

Imamoto, Yasushi; Kojima, Keiichi; Oka, Toshihiko; Maeda, Ryo; Shichida, Yoshinori

\section{CITATION:}

Imamoto, Yasushi ...[et al]. Conformational differences among metarhodopsin I, metarhodopsin II, and opsin probed by wide-angle X-ray scattering. The Journal of Physical Chemistry B 2019, 123(43): 9134-9142

\section{ISSUE DATE:}

2019-10-31

URL:

http://hdl.handle.net/2433/244712

\section{RIGHT:}

This document is the Accepted Manuscript version of a Published Work that appeared in final form in The Journal of Physical Chemistry B, copyright (C) American Chemical Society after peer review and technical editing by the publisher To access the final edited and published work see https://doi.org/10.1021/acs.jpcb.9b08311.; The full-text file will be made open to the public on 3 October 2020 in accordance with publisher's 'Terms and Conditions for Self-Archiving'.: This is not the published version. Please cite only the published version.; この論文は出版社版でありません。引用の際 には出版社版をご確認ご利用ください。 


\section{Conformational Differences among Metarhodopsin I,}

\section{Metarhodopsin II, and Opsin Probed by Wide-Angle}

\section{X-ray Scattering}

Yasushi Imamoto, ${ }^{*}{ }^{\dagger}$ Keiichi Kojima, ${ }^{\dagger}$ Toshihiko Oka, ${ }^{\|,+}$Ryo Maeda, ${ }^{\dagger}$ and Yoshinori Shichida ${ }^{\S}$

†Department of Biophysics, Graduate School of Science, Kyoto University, Kyoto, Japan,

"Department of Physics, Faculty of Science and Nanomaterials Research Division, Research Institute of Electronics, Shizuoka University, Shizuoka, Japan, and ${ }^{\S}$ Research Organization for Science and Technology, Ritsumeikan University, Kusatsu, Shiga, Japan. 
ABSTRACT: Among the photoproducts of vertebrate rhodopsin, only metarhodopsin II (MetaII) preferentially adopts the active structure in which transmembrane helices are rearranged. Light-induced helical rearrangement of rhodopsin in membrane-embedded form was directly monitored by wide-angle X-ray scattering (WAXS) using nanodiscs. The change in WAXS curve for the formation of Meta-II was characterized by peak at $0.2 \AA^{-1}$ and valley at $0.6 \AA^{-1}$, which were not observed in metarhodopsin I and opsin. However, acid-induced active opsin $\left(\right.$ Opsin $\left.{ }^{*}\right)$ showed $0.2 \AA^{-1}$ peak but no $0.6 \AA^{-1}$ valley. Analyses using the model structures based on the crystal structures of dark state and Meta-II suggest that the outward movement of helix VI occurred in Opsin*. However, the displaced helices III and V in Meta-II resulting from the disruption of cytoplasmic ionic lock were restored in Opsin ${ }^{*}$, which is likely to destabilize the Gprotein activating structure of opsin. 


\section{INTRODUCTION}

Vertebrate rhodopsins are well-characterized G-protein coupled receptors (GPCRs), which transmit the extracellular signal to initiate intracellular signal by activating cognate G-proteins. Because GPCRs are the most important pharmacological target, its activation and deactivation mechanisms are essential for drug design. Rhodopsin and GPCRs share a common seven transmembrane structural motif ${ }^{1-2}$. In response to stimuli, the transmembrane helices are rearranged, and the cytoplasmic G-protein binding site is activated. While binding of the diffusible ligand stimulates GPCRs in general, stimulus to rhodopsin is the photoisomerization of the 11-cis-retinal chromophore, resulting in the generation of an agonist all-trans-retinal chromophore in the chromophore-binding site.

The photobleaching process of rhodopsin has been extensively studied, and several intermediates have been identified ${ }^{3}$. While recent work using time-resolved absorption techniques under physiologically relevant conditions showed the branched scheme involving several additional intermediates, ${ }^{4}$ the classical simple model well-describes the photobleaching process of rhodopsin (Figure 1). In the dark state rhodopsin, the 11-cis-retinal chromophore covalently binds to Lys296 via a protonated Schiff base. On photon absorption, the chromophore is isomerized to all-trans-retinal and the primary intermediate, photorhodopsin (Photo) is formed. Concomitant with the relaxation of the twisted chromophore, the structural changes in the protein moiety takes place, and bathorhodopsin (Batho), lumirhodopsin (Lumi), and metarhodopsin I (Meta-I) are successively formed. After Meta-I, the chromophore Schiff base is deprotonated and G protein-activating intermediate, metarhodopsin II (Meta-II) is formed. Meta-II decays into 
apoprotein opsin by the hydrolysis of the Schiff base, or into relatively stable metarhodopsin III (Meta-III).

In the dark state rhodopsin, 11-cis-retinal acts as an inverse agonist, which substantially reduces the G-protein activation efficiency ${ }^{5}$. The ionic lock between protonated Schiff base and the nearby counterion (Glu113 in bovine rhodopsin) cooperatively maintain the inactive structure with cytoplasmic ionic lock between helices III and VI ${ }^{6}$. In Meta-II, deprotonation of Schiff base disrupts the ionic lock, resulting in the substantial rearrangement of transmembrane helices ${ }^{7-9}$. In opsin, all-trans-retinal, the agonist of Meta-II, is released by the hydrolysis of the Schiff base. The significantly lower G-protein activation efficiency of opsin than Meta-II as well as the spectroscopic analysis ${ }^{10-13}$ strongly suggests that the helical arrangement reverts to the closed structure in opsin. However, the crystal structure of opsin formed at acidic $\mathrm{pH}$ adopts the active structure indistinguishable from Meta-II ${ }^{14-16}$. While opsin adopts Meta-II-like structure at low $\mathrm{pH}^{12}$ (Figure 1), these findings raise the question of whether crystal structure of Opsin ${ }^{*}$ (3CAP ${ }^{14}$ ) serves as the model of physiologic opsin.

The structural information of Meta-II and its relevant intermediates are essential to understand the regulation mechanism of G-protein activation by photoactivated rhodopsin. Our previous study using single-molecule fluorescence spectroscopy demonstrated that rhodopsin (opsin) molecule is intrinsically in equilibrium between active (open) and inactive (closed) conformations, and the equilibrium could be shifted to either open or closed conformation depending on the existence, isomerization state, or protonation state of the chromophore (ligand) 17-18. Among dark state, intermediate states, and opsin state of rhodopsin, open conformation is dominant only in Meta-II. To reveal the ligand-induced activation mechanism of rhodopsin, it is important to demonstrate the conformational difference in open structures between Meta-II and 
other states. For direct observations of the helical rearrangements in physiological condition, we have performed the wide-angle (high-angle) X-ray scattering measurements (WAXS) using nanodiscs (ND) ${ }^{19}$.

In WAXS experiments, the scattering intensity of incident X-ray in wider angle region than Guinier region is measured. Momentum transfer $(Q=4 \pi \sin \theta / \lambda$, where $\lambda$ is the wavelength of the incident X-ray) ranging from 0.1 to $1.0 \AA^{-1}$ corresponds to real-space distance $(D=2 \pi / Q)$ ranging from 63 to $6.3 \AA$, which reports the situation of the packing of secondary structure elements ${ }^{19-23}$. We have demonstrated that the light-induced structural change of membrane-embedded rhodopsin is similar to that demonstrated by the crystal structure of Meta-II ${ }^{19}$. In the current study, changes in WAXS curves (difference WAXS curves) for formation of Meta-I, inactive opsin $\left(O p \sin ^{-}\right)$, and acid-induced active opsin $\left(O p \sin ^{*}\right)$ were measured in addition to Meta-II. The results demonstrated that the difference WAXS curve for Opsin ${ }^{*}$ formation was different from that of Meta-II, as opposed to the deduction from crystallographic Opsin" structure (3CAP $\left.{ }^{14}\right)$. The model structures based on the crystal structures of dark state and Meta-II suggested that the outward movement of helix VI takes place in Opsin*, but the displacement of cytoplasmic side of helices III and V in Meta-II relative to dark state is restored in Opsin*. In Meta-II, helix III interacts with helix V via conserved D(E)RY motif and Tyr223 ${ }^{16}$, and constitutively active mutation site Met257 is close to this region. It is likely that the restoration of the cytoplasmic ionic lock, which is coupled with the hydrogen bond between Glu113 and Lys296, destabilizes the active conformation in opsin.

\section{MATERIALS AND METHODS}


Bovine rhodopsin was isolated from bovine retina and incorporated into ND as reported previously $19,24-26$. Briefly, rhodopsin solubilized with $1 \%$ n-octyl- $\beta$-D-glucoside (OG) was mixed with membrane scaffold protein (MSP) MSP1E3D1 and 1-palmitoyl-2-oleoyl-sn-glycero3-phosphocholine (POPC) at a molar ratio of $750: 10: 1$ (lipids : MSP : pigment). ND was selfassembled through the removal of OG by dialysis. Large molar excess of MSP and lipid over rhodopsin yields NDs containing one or no rhodopsin molecule. ND containing rhodopsin (Rh/ND) was isolated through Superdex 200 column chromatography, followed by concanavalin A affinity column chromatography. The stoichiometry of rhodopsin and MSP was estimated to be $1: 2$ by absorbance at $500 \mathrm{~nm}$ and $278 \mathrm{~nm}$, indicating that ND contained one rhodopsin molecule. Empty ND for the control experiments was prepared with MSP and lipids.

ND was suspended in HEPES buffer (50 mM HEPES, $140 \mathrm{mM} \mathrm{NaCl}, \mathrm{pH}$ 8.0) for formation of Meta-I, citrate buffer (50 mM sodium citrate, $140 \mathrm{mM} \mathrm{NaCl}, \mathrm{pH} 4.0)$ containing $100 \mathrm{mM}$ hydroxylamine for formation of Opsin* or MES buffer (50 mM MES, $140 \mathrm{mM} \mathrm{NaCl}, \mathrm{pH} 6.0$ ) containing $100 \mathrm{mM}$ hydroxylamine for formation of Opsin- ${ }^{-}$For the formation of Meta-II, $\mathrm{Rh} / \mathrm{ND}$ was suspended in MES buffer or citrate buffer containing no hydroxylamine. $\mathrm{Rh} / \mathrm{ND}$ was concentrated to $5-15 \mathrm{mg}$ rhodopsin/mL using an ultrafiltration membrane.

WAXS was measured at the beam line BL40B2 in SPring-8 (Sayo, Japan). The sample was put into the quartz capillary of $2 \mathrm{~mm}$ in diameter (Hilgenberg 4017250), and illuminated from the top of the sample cell holder using fiber light source, equipped with glass cutoff filter (Edmund OG515, >500 nm) to photoactivate rhodopsin. For formation of Opsin' ${ }^{-}$and Opsin*, WAXS measurements were started $7-10 \mathrm{~min}$ after illumination. The temperature of the sample cell holder was maintained at $20^{\circ} \mathrm{C}$ by circulating the temperature-controlled water. The concentric scattering image was obtained by R-AXIS VII (Rigaku), with detector surface at $540 \mathrm{~mm}$ from 
the sample. To reduce radiation damage, the $25 \%$ attenuator was set in the path of the X-ray, and sample stage was moved to and fro along the capillary axis. The sample was exposed to X-ray for $1 \mathrm{~min}$ to obtain one scattering image, and it took about 2 min for readout. The WAXS curve ( $I$ vs $Q$ ) was calculated by circularly averaging the scattering image. The measurements were repeated 8 times, and rhodopsin was photoactivated between the fourth and the fifth measurements. To eliminate the possible effect of X-ray damage, the difference WAXS curves were calculated using the extrapolated curves just before the photoactivation derived from the first to fourth measurements, and just after the photoactivation derived from the fifth to eighth measurements ${ }^{19}$. These data sets were obtained 2-7 times and averaged curves are presented. After WAXS measurements, hydroxylamine was added to the sample, and the amount of photoreactivated rhodopsin was determined by UV-visible spectrophotometer (Shimadzu UV2400PC) to scale the difference WAXS curves.

\section{RESULTS}

\section{Detection of light-induced helical rearrangement by WAXS.}

We have performed WAXS measurements using ND to assess the conformational change of rhodopsin in membrane-embedded form. In the current work, WAXS measurements were applied to Meta-I, opsin in inactive form $\left(\mathrm{Opsin}^{-}\right)$, and opsin in active form (Opsin $\left.{ }^{*}\right)$ in addition to Meta-II (Figure 2a). Because Meta-I and Meta-II are in pH-dependent equilibrium ${ }^{27-28}$, $\mathrm{Rh} / \mathrm{ND}$ was suspended in buffer at $\mathrm{pH} 8.0$ and $\mathrm{pH} 6.0$ for the predominant formations of Meta-I and Meta-II, respectively (75-80\%). To generate opsin, Rh/ND was suspended in buffer containing $100 \mathrm{mM}$ hydroxylamine, which readily converts Meta-I/Meta-II into opsin and alltrans-retinal oxime (Figure S1). Because Opsin ${ }^{-}$and Opsin ${ }^{*}$ are in $\mathrm{pH}-$ dependent equilibrium ${ }^{12}$, 
$\mathrm{Rh} / \mathrm{ND}$ was suspended in buffer at $\mathrm{pH} 6.0$ and $\mathrm{pH} 4.0$ for the predominant formation of Opsin ${ }^{-}$ and Opsin", respectively. The difference WAXS curve of Meta-II was also measured at pH 4.0 for comparison. Because WAXS curves of ND are highly sensitive to X-ray radiation ${ }^{19}$, the stability of the baseline against X-ray radiation and visible light irradiation was assessed by measuring empty ND in the same condition. The difference WAXS curve is presented as $\Delta I \times Q$ vs $Q$ in this work to emphasize the intensity difference in wide angle region, which possibly reports the helical rearrangements. The difference WAXS curves in $\Delta I$ vs $Q$ are shown in Figure S2.

The difference WAXS curve showed that at $\mathrm{pH}$ 6.0, where Meta-II is predominantly formed, there was a sharp valley at $Q=0.13 \AA^{-1}$, sharp peak at $Q=0.22 \AA^{-1}$, and broad valley at $Q=0.60 \AA^{-1}$. In DDM micelle, the intensity change at $Q=0.22 \AA^{-1}$ was smaller than that in ND, but valley at $Q=0.60 \AA^{-1}$ was similarly observed ${ }^{19}$.

At $\mathrm{pH}$ 8.0, where Meta-I is predominantly formed, the small valley was observed at $Q=0.14 \AA^{-}$ ${ }^{1}$ but peak at $Q=0.22 \AA^{-1}$ and valley at $Q=0.60 \AA^{-1}$ were absent, suggesting that the helical rearrangement of Meta-I is substantially smaller than that of Meta-II. In the presence of $100 \mathrm{mM}$ hydroxylamine at $\mathrm{pH}$ 6.0, the difference WAXS curve for Opsin ${ }^{-}$was comparable to that of Meta-I, whereas broad positive band was observed at $Q=0.2-0.6 \AA^{-1}$.

In the presence of $100 \mathrm{mM}$ hydroxylamine at $\mathrm{pH} 4.0$, a sharp valley at $Q=0.13 \AA$ and sharp peak at $Q=0.22 \AA^{-1}$ were observed for Opsin ${ }^{*}$ like Meta-II. However, valley at $Q=0.60 \AA^{-1}$ was absent in Opsin ${ }^{*}$ unlike Meta-II at pH 6.0. To assess whether this difference is derived from the difference in $\mathrm{pH}$, the difference WAXS curve for Meta-II at $\mathrm{pH} 4.0$ was measured. The result demonstrated that the valley at $Q=0.60 \AA^{-1}$ was also present in difference WAXS curves for Meta-II at pH 4.0. 
Previous FTIR experiment demonstrated that $\mathrm{p} K$ between Opsin${ }^{-}$and $O p \sin ^{*}$ is 4.1-4.3 ${ }^{12}$, suggesting that only half of photoproduct is Opsin* at $\mathrm{pH} 4$. However, the peak intensity at $Q=0.2 \AA^{-1}$ of difference WAXS curve for Opsin ${ }^{*}$ was comparable to that for Meta-II at $\mathrm{pH} 4$, indicating that the amount of $\mathrm{Opsin}^{-}$is small in our experimental condition. $\mathrm{p} K$ between Opsin ${ }^{-}$ and Opsin ${ }^{*}$ in ND in buffer solution would be greater than that in hydrated film for FTIR measurements. It should be noted that the difference WAXS curve for formation of the mixture of Opsin', Opsin*, and potentially another unidentified state should be the linear combination of pure difference WAXS curves of them. Therefore, if difference WAXS curve for the predominant acid-induced component (Opsin $\left.{ }^{*}\right)$ is identical to that for Meta-II, peak at $Q=0.2 \AA^{-1}$ and valley at $Q=0.6 \AA^{-1}$ should be weakened simultaneously. Taken together, it is likely that the absence of the valley at $Q=0.6 \AA^{-1}$ is derived from the different helical arrangement of Opsin* from that of Meta-II.

So far, conformational changes of rhodopsin molecule have been studied through X-ray crystallography. To show the relationship between WAXS difference and conformational change, the difference WAXS curves were deduced from the crystal structures of Batho (2G87) ${ }^{29}$, Lumi $(2 \mathrm{HPY}){ }^{30}$, deprotonated intermediate (2I37) ${ }^{31}$, Meta-II (3PXO) ${ }^{16}$, and Opsin ${ }^{*}$ (3CAP) ${ }^{14}$ using CRYSOL, which is a well-established program for evaluating the experimental solution X-ray scattering curves of macromolecules using known atomic structure. ${ }^{32}$ (Figure $2 \mathrm{~b}$ and Table S1). The high-resolution structures of Meta-I and Opsin ${ }^{-}$have not been available until now.

The difference WAXS curve between dark state and Batho (Batho/Dark) is almost flat, indicating that the conformational difference between them is not detectable by WAXS measurement. Meanwhile, valley at $Q=0.1 \AA^{-1}$ and broad peak at $Q=0.3-0.7 \AA^{-1}$ were observed for Lumi (Lumi/Dark). The crystal structure of Lumi indicates the local displacement of the 
peptide near the chromophore ${ }^{30}$, suggesting that WAXS is sensitive to local changes. This calculated curve is similar to experimental curves for Meta-I/Dark and Opsin-/Dark.

The crystal structure of Meta-II (3PXO) gave substantially greater difference WAXS curves than those of Lumi (2HPY) and deprotonated intermediate (2I37). The difference WAXS curve derived from the crystal structure of Opsin* (3CAP) was very similar to that of Meta-II.

The experimental (Meta-II/Dark) and calculated (3PXO/1U19) difference WAXS curves for G-protein activating conformation are characterized by sharp valley at $Q=0.1 \AA^{-1}$, sharp peak at $Q=0.2 \AA^{-1}$ and broad valley at $Q=0.6 \AA^{-1}$ (Figure 2). This tendency is also observed when other crystal structures of dark state and Meta-II were used (1GZM, 2X72, and 4A4M, Figure S3 and Table S1). However, experimental curve for Opsin ${ }^{*}$ showed valley at $Q=0.1 \AA^{-1}$ and peak at $Q=0.2 \AA^{-1}$, but no valley at $Q=0.6 \AA^{-1}$ as opposed to crystallographic Opsin* (3CAP) (Figures 2). Meanwhile, experimental curves for Meta-I and Opsin- showed valley at $Q=0.1 \AA^{-1}$, whereas Opsin $^{-}$showed a broad peak at $Q=0.2-0.6 \AA^{-1}$ similar to Lumi (2HPY/1U19). Therefore, the identification of the origins of these features would provide the information about the conformational difference between these states.

\section{Structural changes that induce scattering changes}

To identify the region of structural change responsible for the scattering intensity changes, the model structures were generated using the crystal structures of dark state (1U19), Lumi (2HPY), and Meta-II (3PXO), and the difference WAXS curves were deduced by CRYSOL as follows:

The PDB files contain the atoms for amino acid residues and heteroatoms such as water, lipids, and retinal. From 1U19 and 3PXO, the atoms for amino acid residues 1-326 and retinal were extracted. These structures were overlapped using the "align" method of PyMol software 
(https://pymol.org) for all atoms. Then secondary structure elements, helices I-VIII (H1-H8) and the second extra cellular loop (ECL2), were interchanged between 1 U19 and 3PXO (Figures 3 and Table S2). No energy minimization was carried out. For example, when helix VI (H6) of $1 \mathrm{U} 19$ was replaced by that of $3 \mathrm{PXO}$, the structure formed was referred to as $3 \mathrm{PXO}^{\mathrm{H} 6}$ (Figure 3a). When $\mathrm{H} 6$ of $3 \mathrm{PXO}$ was replaced with that of 1U19, the structure formed had 3PXO structure except for H6. Thus, it was referred to as $3 \mathrm{PXO}^{\overline{\mathrm{H} 6}}$ (Figure $3 \mathrm{~b}$ ). The WAXS curves of these model structures were calculated by CRYSOL, from which the WAXS curve of 1 U19 was subtracted, and multiplied by $Q$. Figure $3 \mathrm{c}$ shows the difference WAXS curves in which the secondary structure element of $1 \mathrm{U} 19$ was replaced with that of $3 \mathrm{PXO}$, whereas Figure $3 \mathrm{~d}$ shows those in which the secondary structure element of $3 \mathrm{PXO}$ was replaced with that of $1 \mathrm{U} 19$. The results demonstrated that the peak at $Q=0.2 \AA^{-1}$ was generated in $3 \mathrm{PXO}^{\mathrm{H} 6} / 1 \mathrm{U} 19$. Reversely, the peak at $Q=0.2 \AA^{-1}$ was significantly reduced in $3 \mathrm{PXO}^{\overline{\mathrm{H} 6}} / 1 \mathrm{U} 19$. Similar effect was also observed when the intracellular half of $\mathrm{H6}(\mathrm{H} 6 \mathrm{IC})$ was substituted $\left(3 \mathrm{PXO}^{\mathrm{H} 6 \mathrm{IC}} / 1 \mathrm{U} 19\right.$ and $\left.3 \mathrm{PXO}^{\overline{\mathrm{H} 6 \mathrm{IC}}} / 1 \mathrm{U} 19\right)$. Therefore, the peak at $Q=0.2 \AA^{-1}$ is mainly attributable to the outward movement of H6.

Compared to the dark state (1U19), H5 of Meta-II (3PXO) is elongated at the cytoplasmic side because of helix formation of N-terminal 12 amino acid residues of H5-H6 loop. In fact, it was demonstrated that there would be great displacement of $\mathrm{C} \alpha$ in this region by the crystal structures (Figure 3e). While the effect of the substitution of H5 alone on the difference WAXS curve was not clear $\left(3 \mathrm{PXO}^{\mathrm{H} 5} / 1 \mathrm{U} 19\right.$ and $\left.3 \mathrm{PXO}^{\overline{\mathrm{H} 5}} / 1 \mathrm{U} 19\right)$, small valley was generated at $Q=0.6 \AA^{-}$ ${ }^{1}$ in $3 \mathrm{PXO}^{\mathrm{H} 5} / 1 \mathrm{U} 19$, and valley of $3 \mathrm{PXO} / 1 \mathrm{U} 19$ at $Q=0.6 \AA^{-1}$ was weak in $3 \mathrm{PXO}^{\overline{\mathrm{H} 5}} / 1 \mathrm{U} 19$. Similar tendency of valley at $Q=0.6 \AA^{-1}$ was observed for the substitution of $\mathrm{H} 3\left(3 \mathrm{PXO}^{\mathrm{H} 3} / 1 \mathrm{U} 19\right.$ and $\left.3 \mathrm{PXO}^{\overline{\mathrm{H}}} / 1 \mathrm{U} 19\right)$. 
The simultaneous replacements of $\mathrm{H} 3$ and $\mathrm{H} 5$ of $1 \mathrm{U} 19\left(3 \mathrm{PXO}^{\mathrm{H} 3 \& \mathrm{H} 5} / 1 \mathrm{U} 19\right)$ generated the valley at $Q=0.6 \AA^{-1}$ comparable to that of $3 \mathrm{PXO} / 1 \mathrm{U} 19$. It was also observed in $3 \mathrm{PXO}^{\mathrm{H} 3 \& \mathrm{HEC}} / 1 \mathrm{U} 19$, where the substitution of helix-forming 12 amino acid residues of H5-H6 loop was not included (Table S2). Therefore, it is likely that valley at $Q=0.6 \AA^{-1}$ is not attributable to the elongation of $\mathrm{H} 5$. Because the replacements of $\mathrm{H} 3$ and $\mathrm{H} 5$ synergistically affected the valley at $0.6 \AA^{-1}$, alternation of the spatial relationship between $\mathrm{H} 3$ and $\mathrm{H} 5$ would be responsible for this intensity change. To examine this idea, the contact region of $\mathrm{H} 3$ and $\mathrm{H} 5$ (cytoplasmic sides of $\mathrm{H} 3$ and $\mathrm{H} 5$, Table $\mathrm{S} 2$ ) was replaced $\left(3 \mathrm{PXO}^{\mathrm{H} 3=\mathrm{H} 5} / 1 \mathrm{U} 19\right.$ and $3 \mathrm{PXO}^{\overline{\mathrm{H} 3=\mathrm{H} 5}}$ /1U19). The results were similar to those of $3 \mathrm{PXO}^{\mathrm{H} 3 \& \mathrm{H} 5} / 1 \mathrm{U} 19$ and $3 \mathrm{PXO}^{\overline{\mathrm{H} 3 \& \mathrm{H}}} / 1 \mathrm{U} 19$, indicating that the valley at $0.6 \AA^{-1}$ was generated by the displacements of cytoplasmic sides of $\mathrm{H} 3$ and $\mathrm{H} 5$. $3 \mathrm{PXO}^{\overline{\mathrm{H} 3=\mathrm{H} 5}} / 1 \mathrm{U} 19$ well reproduced the difference WAXS curve for Opsin ${ }^{*}$ measured at $\mathrm{pH} 4.0$.

The difference WAXS curve for the formation of Lumi deduced from the crystal structure (2HPY) was substantially smaller than that of Meta-II (Figure 2b), but it was partially similar to the experimental curves for Meta-I and Opsin'. Therefore, similar analyses using the hybrid structures of dark state (1U19) and Lumi (2HPY) were carried out to assess the structural change in Meta-I and Opsin- (Figures 4 and Table S3). Figure 4c shows the difference WAXS curves in which some secondary structure element of $1 \mathrm{U} 19$ was replaced with that of 2HPY, whereas Figure $4 \mathrm{~d}$ shows those in which secondary structure of 2HPY were replaced with that of 1 U19.

The difference WAXS curve calculated using 1U19 and 2HPY (2HPY/1U19) shows the valley at $Q=0.1 \AA^{-1}$ and broad peak at $Q=0.3-0.6 \AA^{-1}$. Reduced valley between $Q=0.11-0.15 \AA^{-1}$ was observed when H3, H4, H5, and H6 of $1 \mathrm{U} 19$ were replaced with those of 2HPY $\left(2 \mathrm{HPY}^{\mathrm{H} 3} / 1 \mathrm{U} 19\right.$, 2HPY $\mathrm{H}^{\mathrm{H}} / 1 \mathrm{U} 19,2 \mathrm{HPY} \mathrm{H} / 1 \mathrm{U} 19$, and $2 \mathrm{HPY}^{\mathrm{H} 6} / 1 \mathrm{U} 19$, respectively), whereas the decrease was negligible for $\mathrm{H} 1, \mathrm{H} 2, \mathrm{H} 7, \mathrm{H} 8$, and ECL2 (2HPY $\mathrm{H}^{\mathrm{H}} / 1 \mathrm{U} 19,2 \mathrm{HPY}^{\mathrm{H} 2} / 1 \mathrm{U} 19,2 \mathrm{HPY}^{\mathrm{H} 7} / 1 \mathrm{U} 19$, 
2HPY ${ }^{\mathrm{H} 8} / 1 \mathrm{U} 19$, and 2HPY ECL2/1U19, respectively). When H3, H4, H5, and H6 of $1 \mathrm{U} 19$ were simultaneously replaced with those of $2 \mathrm{HPY}\left(2 \mathrm{HPY}^{\mathrm{H} 3-\mathrm{H} 6} / 1 \mathrm{U} 19\right)$, the intensity decrease was comparable to that of 2HPY/1U19. Inversely, when H3, H4, H5 and H6 of 2HPY were simultaneously replaced with those of $1 \mathrm{U} 19\left(2 \mathrm{HPY}^{\overline{\mathrm{H} 3-\mathrm{H} 6}} / 1 \mathrm{U} 19\right)$, the intensity decrease was substantially reduced. These observations demonstrate that the valley at $Q=0.1 \AA^{-1}$ in 2HPY/1U19 is originated from the structural changes in $\mathrm{H} 3, \mathrm{H} 4, \mathrm{H} 5$ and $\mathrm{H} 6$, which form the chromophore binding site.

2HPY/1U19 showed the characteristic shoulder at $Q=0.6 \AA^{-1}$. This shoulder was also observed in both of $2 \mathrm{HPY} \mathrm{H}^{\mathrm{H}-\mathrm{H} 6} / 1 \mathrm{U} 19$ and $2 \mathrm{HPY} \overline{\mathrm{H3}-\mathrm{H} 6} / 1 \mathrm{U} 19$, while the effect of substitution of $\mathrm{H} 4$ is the largest. Therefore, the shoulder at $Q=0.6 \AA^{-1}$ in $2 \mathrm{HPY} / 1 \mathrm{U} 19$ is likely to reflect small but global rearrangement of the helices around the chromophore (Figure 4e). It should be noted that this shoulder was not apparent in Meta-I and reduced in Opsin-. Therefore, the helical arrangement of Meta-I is almost similar to that of the dark state, whereas that of Opsin ${ }^{-}$is likely to be in between those of crystallographic dark state and Lumi.

\section{DISCUSSION}

We have performed WAXS measurements using ND to detect the light-induced helical rearrangements of rhodopsin in membrane-embedded form. The results demonstrated that the difference WAXS curve for Meta-II of monomeric rhodopsin in ND well agreed with that calculated from the crystal structure of Meta-II (3PXO), indicating that WAXS is a powerful tool to detect the helical rearrangement of rhodopsin and possibly other GPCRs.

By using CRYSOL software, the difference WAXS curves are anticipated based on the crystal structures of dark state and intermediate states. The crystal structures of dark state (1U19) and 
Batho (2G87) gave a negligible scattering profile change. This agreement in the WAXS curve indicates that the isomerization of chromophore has little contribution to whole WAXS curve. Meanwhile, significant decrease in scattering intensity at $Q=0.1 \AA^{-1}$ was anticipated by the crystal structure of Lumi (2HPY). The crystal structure of Lumi demonstrates that the helices around the chromophore are slightly rearranged in Lumi, indicating that WAXS is sensitive enough to detect small rearrangement in helices.

The difference WAXS curve upon irradiation of $\mathrm{Rh} / \mathrm{ND}$ at $\mathrm{pH} 8.0$, where Meta-I is predominantly formed, was comparable to that deduced from the crystal structures of Lumi and dark state using CRYSOL. This is consistent with the finding of FTIR spectroscopy, in which the difference FTIR spectra of Meta-I/Dark in amide I region is comparable to that of Lumi/Dark ${ }^{33-}$ ${ }^{34}$. While FTIR spectroscopy is sensitive to amide mode, which undergoes the alternation of the hydrogen bond strength on conformational change, WAXS data includes the information of whole arrangement of helices. Therefore, the present findings convincingly demonstrate that the closed conformation is maintained up to Meta-I in the membrane. The difference WAXS curve for Meta-I is explained by the local peptide movements of H3-H6 forming the chromophore binding site (Figure 4), which is consistent with the structure of Meta-I proposed by electron microscope using two dimensional crystal ${ }^{35}$.

The peak at $Q=0.22 \AA^{-1}$ in the difference WAXS curve of Opsin ${ }^{*}$ at $\mathrm{pH} 4.0$ shows the outward movement of cytoplasmic side of helix VI. Because constitutive activity of opsin is explained by the population of Opsin ${ }^{* 18}$, it is likely that the outward movement of helix VI is responsible for the activation capability of $\mathrm{G}$ protein transducin $(\mathrm{Gt})$. However, the lack of valley at $Q=0.6 \AA^{-1}$ in Opsin*, as opposed to Meta-II and crystallographic Opsin*, demonstrates that the displaced cytoplasmic sides of H3 and H5 in Meta-II are restored to dark structure in Opsin* in membrane 
(Figure 5). This region contains D(E)RY motif (Glu134-Tyr136) and Tyr223, and proximate to the strong constitutively active mutation site (Met257) ${ }^{18,36}$. In the crystal structure of Meta-II of M257Y rhodopsin, Arg135, Tyr223, and Tyr257 form the cluster ${ }^{37}$, which possibly stabilizes the fully active conformation. Therefore, this region would modulate the conformational equilibrium between active and inactive conformations.

Our recent single molecule fluorescence spectroscopy using fluorescence label at cytoplasmic H8 demonstrated that the structure of each photoproduct of rhodopsin is not uniform ${ }^{17-18}$. Namely, Meta-I, Meta-II, and opsin are individually in the thermal equilibrium between open and closed conformations, which is modulated by existence, isomerization state, or protonation state of the chromophore (ligand). In opsin state, Opsin- (closed) is more stable than Opsin ${ }^{*}$ (open), and $\Delta \mathrm{G}$ between $\mathrm{Opsin}^{-}$and Opsin${ }^{*}$ was estimated to be $+6.5( \pm 0.5) \mathrm{kJ} / \mathrm{mol}{ }^{18}$. Meanwhile, closed structure of Meta-I (Meta-I $\left.{ }^{-}\right)$is more stable than open structure (Meta-I*), whereas open structure of Meta-II (Meta-II') is more stable than closed structure (Meta-II $\left.{ }^{-}\right) . \Delta \mathrm{G}$ between closed and open structures of Meta-I and Meta-II is $+3.5( \pm 0.4) \mathrm{kJ} / \mathrm{mol}$ and $-4.9( \pm 1.2)$ $\mathrm{kJ} / \mathrm{mol}$, respectively ${ }^{17}$. The agonist of Meta-II is the deprotonated all-trans-retinal Schiff base, which stabilizes the open structure of Meta-II by the steric effect of retinal moiety and/or the electrostatic effect by the neutralization of the ionic lock. Assuming that Glu113 and Lys296 in opsin form the ionic lock similar to that of Meta-I, $\Delta \Delta \mathrm{G}$ between Meta-I and opsin $(+3.0 \mathrm{~kJ} / \mathrm{mol})$, which stabilizes inactive conformation in Opsin state compared to Meta-I, accounts for the steric effect of all-trans-retinal. Meanwhile, $\Delta \Delta \mathrm{G}$ between Meta-I and Meta-II (-8.4 kJ/mol), which stabilizes active conformation in Meta-II compared to Meta-I, is derived from the neutralization of the chromophore Schiff base. Therefore, the disruption of chromophore ionic lock has greater 
contribution to the preferential formation of active structure in Meta-II than the steric effect of retinal moiety.

It is proposed that the cytoplasmic ionic lock is coupled with Schiff base ionic lock ${ }^{6}$. While the previous FTIR spectroscopy demonstrated that Glu113 is neutral in Opsin ${ }^{* 12}$, it could form hydrogen bond with Lys296. It is likely that this interaction restores helices III and V in Opsin*, by which opsin preferentially adopts the inactive conformation. The deprotonation of Schiff base linkage of Meta-II, that is the loss of hydrogen-bond-forming hydrogen as opposed to opsin, would be the key factor for the activation of rhodopsin.

\section{ASSOCIATED CONTENT}

Supporting Information. The Supporting Information is available free of charge on the ACS Publications website. Design of hybrid structures of dark state and intermediates, UV-visible spectra on formation of opsin by hydroxylamine, difference WAXS curves presented in $\Delta I$ vs $Q$, and difference WAXS curves deduced from other crystal structures (PDF).

\section{AUTHOR INFORMATION}

\section{Corresponding Author}

*imamoto@rh.biophys.kyoto-u.ac.jp

\section{Author Contributions}

Y. I. and T. O. designed the research. K. K., R. M., and Y. I. prepared the sample. K. K., R. M., T. O. and Y. I. performed the WAXS measurements and analysis. Y. I. wrote the manuscript with contributions from all other authors. 


\section{Notes}

The authors declare no competing financial interest.

\section{ACKNOWLEDGMENT}

This work was supported by The Kyoto University Foundation, and ISHIZUE 2019 of Kyoto University Research Development Program. The synchrotron radiation experiments were performed at the BL40B2 in the SPring-8 with the approval of the Japan Synchrotron Radiation Research Institute (JASRI) (Proposal No. 2012A1263, 2013A1136, 2014A1247, 2016A1427, and 2016B1258). A part of this work was carried out under the Cooperative Research Project Program of Research Institute of Electronics, Shizuoka University.

\section{ABBREVIATIONS}

GPCR, G-protein-coupled receptor; Batho, bathorhodopsin; Lumi, lumirhodopsin; Meta-I, metarhodopsin I; Meta-II, metarhodopsin II; Opsin ${ }^{*}$, opsin in an active conformation; Opsin', opsin in an inactive conformation; WAXS, wide-angle X-ray scattering; ND, nanodisc; MSP, membrane scaffold protein; Gt, rod G protein transducin; $\mathrm{Rh} / \mathrm{ND}$, nanodisc containing rhodopsin. 


\section{REFERENCES}

1. Deupi, X.; Standfuss, J. Structural insights into agonist-induced activation of G-proteincoupled receptors. Curr. Opin. Struct. Biol. 2011, 21, 541-551.

2. Palczewski, K.; Kumasaka, T.; Hori, T.; Behnke, C. A.; Motoshima, H.; Fox, B. A.; Le Trong, I.; Teller, D. C.; Okada, T.; Stenkamp, R. E.; et al. Crystal structure of rhodopsin: A G protein-coupled receptor. Science 2000, 289, 739-745.

3. Shichida, Y.; Imai, H. Visual pigment: G-protein-coupled receptor for light signals. Cell Mol. Life Sci. 1998, 54, 1299-1315.

4. Szundi, I.; Funatogawa, C.; Guo, Y.; Yan, E. C. Y.; Kliger, D. S. Protein sequence and membrane lipid roles in the activation kinetics of bovine and human rhodopsins. Biophys. J. 2017, 113, 1934-1944.

5. $\quad$ Yanagawa, M.; Kojima, K.; Yamashita, T.; Imamoto, Y.; Matsuyama, T.; Nakanishi, K.; Yamano, Y.; Wada, A.; Sako, Y.; Shichida, Y. Origin of the low thermal isomerization rate of rhodopsin chromophore. Sci. Rep. 2015, 5, 11081.

6. Vogel, R.; Sakmar, T. P.; Sheves, M.; Siebert, F. Coupling of protonation switches during rhodopsin activation. Photochem. Photobiol. 2007, 83, 286-292.

7. Van Eps, N.; Caro, L. N.; Morizumi, T.; Kusnetzow, A. K.; Szczepek, M.; Hofmann, K. P.; Bayburt, T. H.; Sligar, S. G.; Ernst, O. P.; Hubbell, W. L. Conformational equilibria of lightactivated rhodopsin in nanodiscs. Proc. Natl. Acad. Sci. U.S.A. 2017, 114, E3268-E3275.

8. $\quad$ Farrens, D. L.; Altenbach, C.; Yang, K.; Hubbell, W. L.; Khorana, H. G. Requirement of rigid-body motion of transmembrane helices for light activation of rhodopsin. Science 1996, 274, 768-770.

9. $\quad$ Yamazaki, Y.; Nagata, T.; Terakita, A.; Kandori, H.; Shichida, Y.; Imamoto, Y. Intramolecular interactions that induce helical rearrangement upon rhodopsin activation: Lightinduced structural changes in metarhodopsin $\mathrm{II}_{\mathrm{a}}$ probed by cysteine $\mathrm{S}-\mathrm{H}$ stretching vibrations. $J$. Biol. Chem. 2014, 289, 13792-13800.

10. Rothschild, K. J.; Gillespie, J.; DeGrip, W. J. Evidence for rhodopsin refolding during the decay of Meta II. Biophys. J. 1987, 51, 345-350.

11. Klinger, A. L.; Braiman, M. S. Structural comparison of metarhodopsin II, metarhodopsin III, and opsin based on kinetic analysis of Fourier transform infrared difference spectra. Biophys $J$ 1992, 63, 1244-1255.

12. Vogel, R.; Siebert, F. Conformations of the active and inactive states of opsin. J. Biol. Chem. 2001, 276, 38487-38493.

13. Katayama, K.; Furutani, Y.; Kandori, H. FTIR study of the photoreaction of bovine rhodopsin in the presence of hydroxylamine. J. Phys. Chem. B 2010, 114, 9039-9046.

14. Park, J. H.; Scheerer, P.; Hofmann, K. P.; Choe, H. W.; Ernst, O. P. Crystal structure of the ligand-free G-protein-coupled receptor opsin. Nature 2008, 454, 183-187.

15. Scheerer, P.; Park, J. H.; Hildebrand, P. W.; Kim, Y. J.; Krauss, N.; Choe, H. W.; Hofmann, K. P.; Ernst, O. P. Crystal structure of opsin in its G-protein-interacting conformation. Nature 2008, 455, 497-502.

16. Choe, H. W.; Kim, Y. J.; Park, J. H.; Morizumi, T.; Pai, E. F.; Krauss, N.; Hofmann, K. P.; Scheerer, P.; Ernst, O. P. Crystal structure of metarhodopsin II. Nature 2011, 471, 651-655.

17. Maeda, R.; Hiroshima, M.; Yamashita, T.; Wada, A.; Nishimura, S.; Sako, Y.; Shichida, Y.; Imamoto, Y. Single-molecule observation of the ligand-induced population shift of rhodopsin, a G-protein-coupled receptor. Biophys. J. 2014, 106, 915-924. 
18. Maeda, R.; Hiroshima, M.; Yamashita, T.; Wada, A.; Sako, Y.; Shichida, Y.; Imamoto, Y. Shift in conformational equilibrium induces constitutive activity of G-protein-coupled receptor, rhodopsin. J. Phys. Chem. B 2018, 122, 4838-4843.

19. Imamoto, Y.; Kojima, K.; Oka, T.; Maeda, R.; Shichida, Y. Helical rearrangement of photoactivated rhodopsin in monomeric and dimeric forms probed by high-angle X-ray scattering. Photochem. Photobiol. Sci. 2015, 14, 1965-1973.

20. Malmerberg, E.; Bovee-Geurts, P. H. M.; Katona, G.; Deupi, X.; Arnlund, D.; Wickstrand, C.; Johansson, L. C.; Westenhoff, S.; Nazarenko, E.; Schertler, G. F. X.; et al. Conformational activation of visual rhodopsin in native disc membranes. Sci. Signal. 2015, 8, ra26.

21. Andersson, M.; Malmerberg, E.; Westenhoff, S.; Katona, G.; Cammarata, M.; Wohri, A. B.; Johansson, L. C.; Ewald, F.; Eklund, M.; Wulff, M.; et al. Structural dynamics of light-driven proton pumps. Structure 2009, 17, 1265-1275.

22. Cammarata, M.; Levantino, M.; Schotte, F.; Anfinrud, P. A.; Ewald, F.; Choi, J.; Cupane, A.; Wulff, M.; Ihee, H. Tracking the structural dynamics of proteins in solution using timeresolved wide-angle X-ray scattering. Nat. Methods 2008, 5, 881-886.

23. Kamikubo, H.; Shimizu, N.; Harigai, M.; Yamazaki, Y.; Imamoto, Y.; Kataoka, M. Characterization of the solution structure of the $\mathrm{M}$ intermediate of photoactive yellow protein using high-angle solution x-ray scattering. Biophys. J. 2007, 92, 3633-3642.

24. Bayburt, T. H.; Leitz, A. J.; Xie, G.; Oprian, D. D.; Sligar, S. G. Transducin activation by nanoscale lipid bilayers containing one and two rhodopsins. J. Biol. Chem. 2007, 282, 1487514881.

25. Bayburt, T. H.; Sligar, S. G. Membrane protein assembly into Nanodiscs. FEBS Lett. 2010, 584, 1721-1727.

26. Kojima, K.; Imamoto, Y.; Maeda, R.; Yamashita, T.; Shichida, Y. Rod visual pigment optimizes active state to achieve efficient $\mathrm{G}$ protein activation as compared with cone visual pigments. J. Biol. Chem. 2014, 289, 5061-5073.

27. Sato, K.; Morizumi, T.; Yamashita, T.; Shichida, Y. Direct observation of the $\mathrm{pH}-$ dependent equilibrium between metarhodopsins I and II and the $\mathrm{pH}$-independent interaction of metarhodopsin II with transducin C-terminal peptide. Biochemistry 2010, 49, 736-741.

28. Zaitseva, E.; Brown, M. F.; Vogel, R. Sequential rearrangement of interhelical networks upon rhodopsin activation in membranes: the Meta $\mathrm{II}_{\mathrm{a}}$ conformational substate. J. Am. Chem. Soc. 2010, 132, 4815-4821.

29. Nakamichi, H.; Okada, T. Crystallographic analysis of primary visual photochemistry. Angew. Chem. Int. Ed. Engl. 2006, 45, 4270-4273.

30. Nakamichi, H.; Okada, T. Local peptide movement in the photoreaction intermediate of rhodopsin. Proc. Natl. Acad. Sci. U. S. A. 2006, 103, 12729-12734.

31. Salom, D.; Lodowski, D. T.; Stenkamp, R. E.; Le Trong, I.; Golczak, M.; Jastrzebska, B.; Harris, T.; Ballesteros, J. A.; Palczewski, K. Crystal structure of a photoactivated deprotonated intermediate of rhodopsin. Proc. Natl. Acad. Sci. U. S. A. 2006, 103, 16123-16128.

32. Svergun, D. I.; Baberato, C.; Koch, M. H. J. CRYSOL-a program to evaluate X-ray solution scattering of biological macromolecules from atomic coordinates. J. Appl. Crystallogr. 1995, 28, 768-773.

33. Maeda, A.; Ohkita, Y. J.; Sasaki, J.; Shichida, Y.; Yoshizawa, T. Water structural changes in lumirhodopsin, metarhodopsin I, and metarhodopsin II upon photolysis of bovine 
rhodopsin: analysis by Fourier transform infrared spectroscopy. Biochemistry 1993, 32, 12033 12038.

34. Nishimura, S.; Sasaki, J.; Kandori, H.; Lugtenburg, J.; Maeda, A. Structural changes in the lumirhodopsin-to-metarhodopsin I conversion of air-dried bovine rhodopsin. Biochemistry 1995, 34, 16758-16763.

35. Ruprecht, J. J.; Mielke, T.; Vogel, R.; Villa, C.; Schertler, G. F. X. Electron crystallography reveals the structure of metarhodopsin I. EMBO J. 2004, 23, 3609-3620.

36. Han, M.; Smith, S. O.; Sakmar, T. P. Constitutive activation of opsin by mutation of methionine 257 on transmembrane helix 6. Biochemistry 1998, 37, 8253-8261.

37. Deupi, X.; Edwards, P.; Singhal, A.; Nickle, B.; Oprian, D.; Schertler, G.; Standfuss, J. Stabilized $\mathrm{G}$ protein binding site in the structure of constitutively active metarhodopsin-II. Proc. Natl. Acad. Sci. U. S. A. 2012, 109, 119-124. 


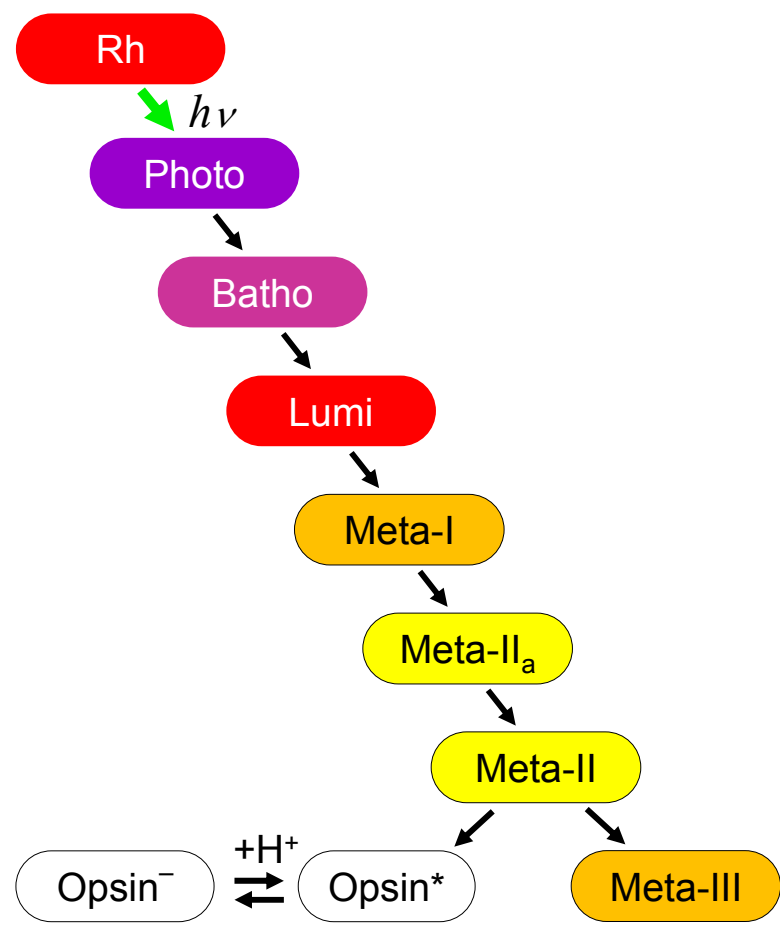

Figure 1: Photobleaching process of vertebrate rhodopsin. 
(a)

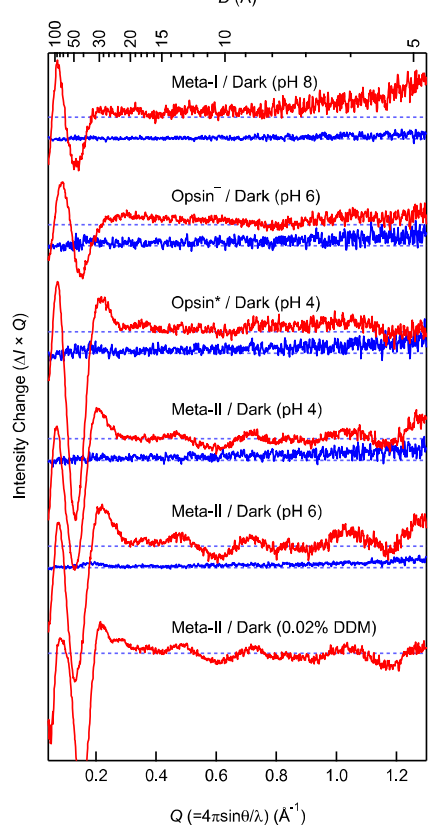

(b)

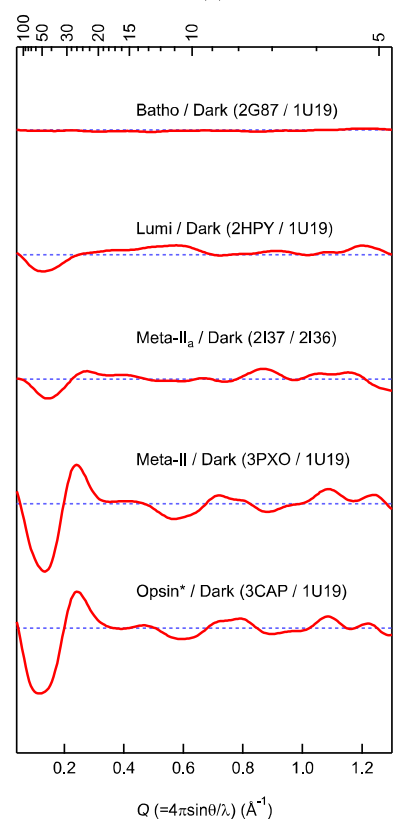

Figure 2: Difference WAXS curves for the formation of photoproducts. (a) Meta-I was generated by irradiation at $\mathrm{pH}$ 8.0. Opsin ${ }^{-}$and Opsin ${ }^{*}$ were generated by irradiation in the presence of 100 $\mathrm{mM}$ hydroxylamine at $\mathrm{pH} 6.0$ and $\mathrm{pH} 4.0$, respectively. Meta-II was produced by irradiation in the absence of hydroxylamine. Meta-II/Dark at pH 6.0 and in DDM micelle were taken from previous paper ${ }^{19}$ for comparison. The stability of the baseline against X-ray radiation and visible light irradiation was assessed by measuring empty ND in the same irradiation conditions (blue lines). Samples were irradiated with yellow light $(>500 \mathrm{~nm})$ for $30 \mathrm{~s}$, and measurements were carried out at $293 \mathrm{~K}$. Presented curves are averages of 2-7 measurements. (b) Difference WAXS curves deduced by crystal structures. The WAXS curves of dark state (1U19 or 2I26), Batho (2G87), Lumi (2HPY), Meta-II (deprotonated intermediate) (2I37), Meta-II (3PXO), and Opsin* (3CAP) were calculated by CRYSOL. Unresolved residues in the photoproducts were deleted from dark state structure (Table S1). 
(a)

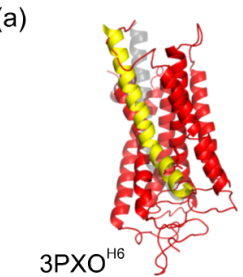

(c)

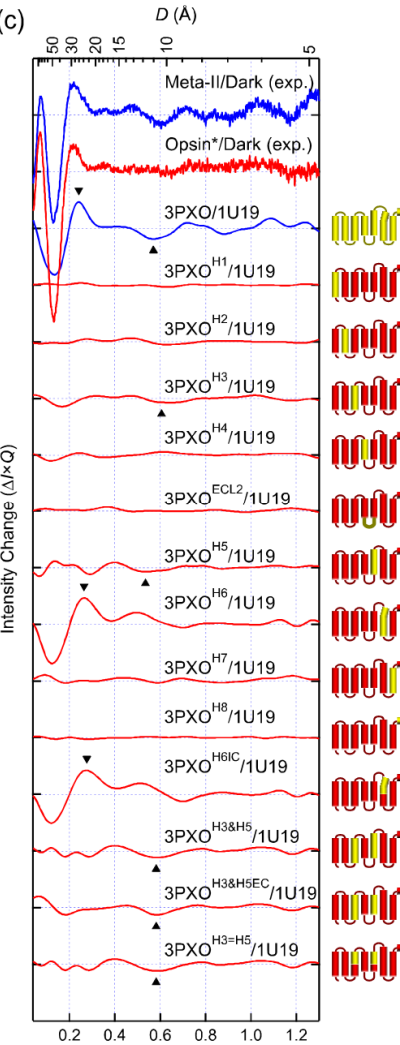

$Q(=4 \pi \sin \theta / \lambda)\left(\AA^{-1}\right)$ (b)

(d)

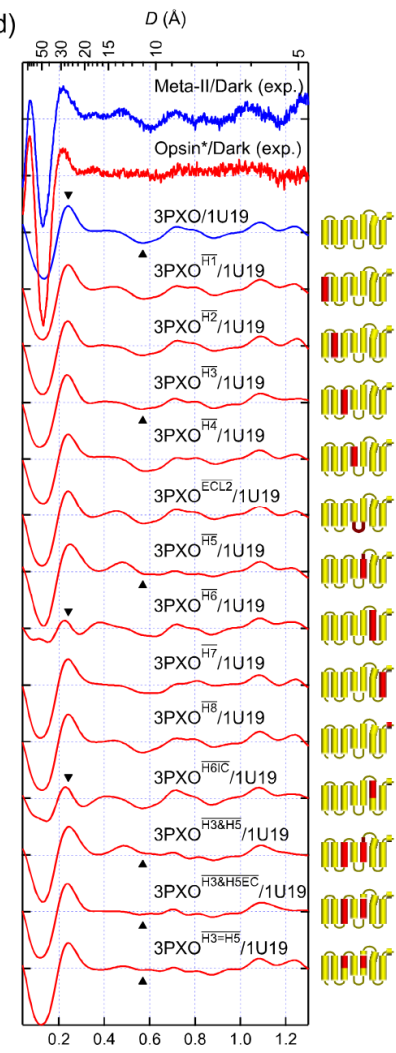

$Q(=4 \pi \sin \theta / \lambda)\left(\AA^{-1}\right)$

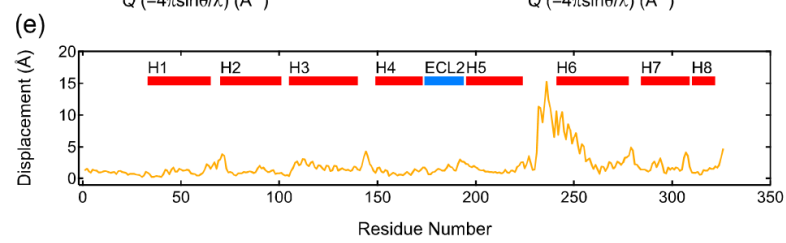

Figure 3: Difference WAXS curves calculated by the hybrid structures of dark state rhodopsin and Meta-II. (a) In the crystal structure of dark-state rhodopsin (1U19, red), atoms for helix VI (gray) were replaced with those of Meta-II (3PXO, yellow). This structure is referred to as $3 \mathrm{PXO}^{\mathrm{H} 6}$. (b) In the crystal structure of Meta-II (3PXO, yellow), atoms for helix VI (gray) were replaced with those of dark-state rhodopsin $\left(1 \mathrm{U} 19\right.$, red). This structure is referred to as $3 \mathrm{PXO}^{\overline{\mathrm{H}} 6}$. 
(c, d) Difference WAXS curves for hybrid structures calculated by CRYSOL. The schematic structures are shown in the figure above (for detail, Table S2). (e) Distances between C $\alpha$ of dark state (1U19) and Meta-II (3PXO) were plotted against the residue number. 
(a)

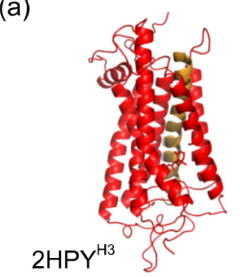

(c) $D(A)$

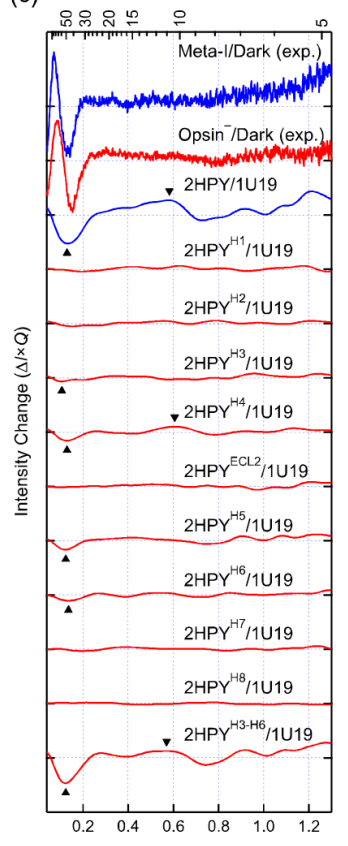

$Q(=4 \pi \sin \theta / \lambda)\left(\AA^{-1}\right)$ (b)

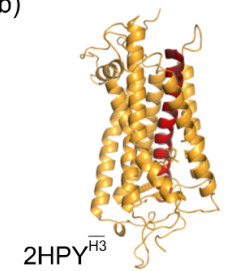

(d)
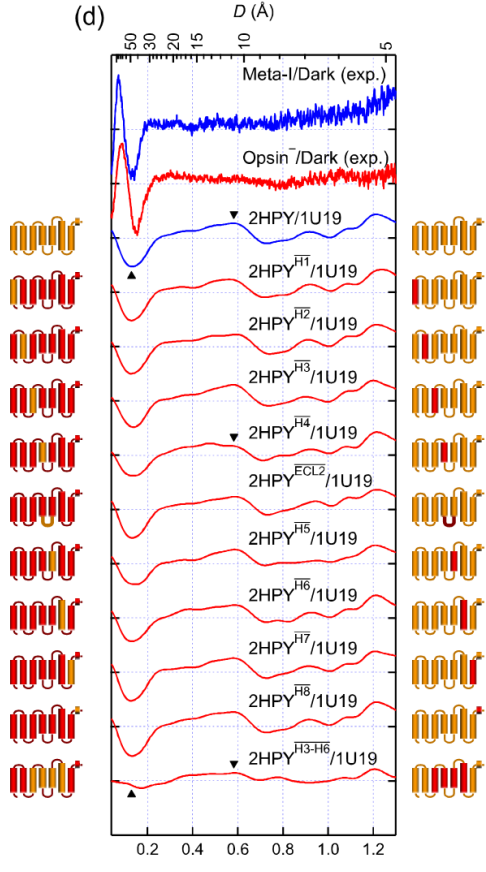

$Q(=4 \pi \sin \theta / \lambda)\left(\AA^{-1}\right)$

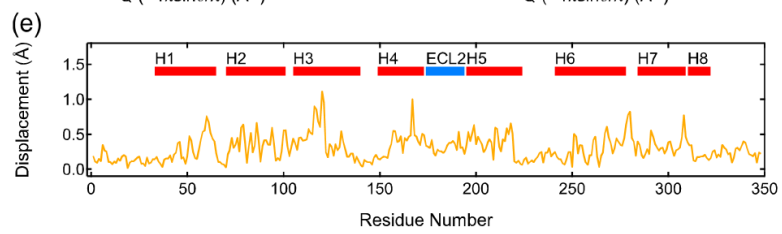

Figure 4: Difference WAXS curves calculated by the hybrid structures of dark state rhodopsin and Lumi. (a) In the crystal structure of dark-state rhodopsin (1U19, red), atoms for helix III (gray) were replaced with those of Lumi (2HPY, orange). This structure is referred to as $2 \mathrm{HPY}^{\mathrm{H} 3}$. (b) In the crystal structure of Lumi (2HPY, orange), atoms for helix III (gray) were replaced with those of dark-state rhodopsin $\left(1 \mathrm{U} 19\right.$, red). This structure is referred to as $2 \mathrm{HPY}^{\overline{\mathrm{H}} 3}$. (c,d) Difference WAXS curves for hybrid structures calculated by CRYSOL. The schematic structures 
are shown in the figure above (for detail, Table S3). (e) Distances between C $\alpha$ of dark state (1U19) and Lumi (2HPY) were plotted against the residue number. 


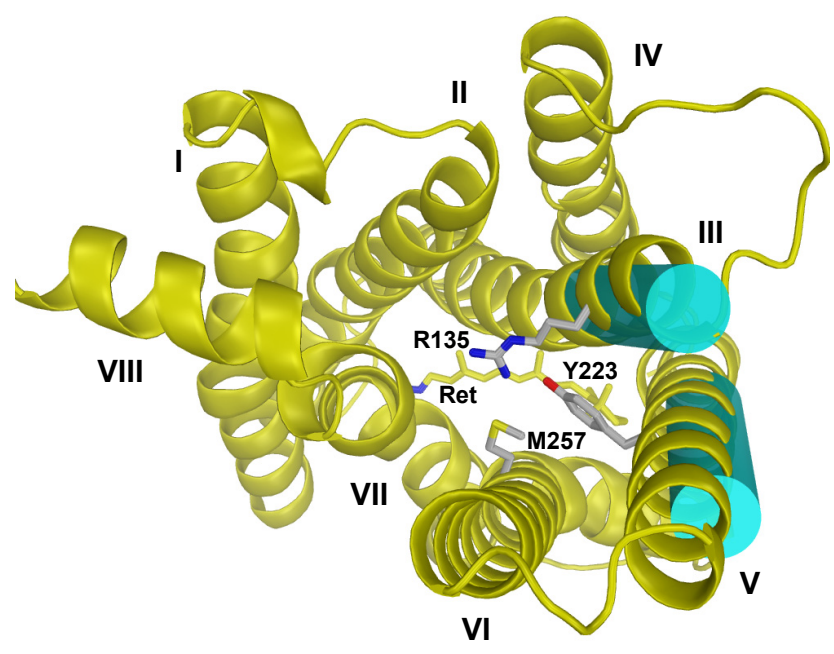

Figure 5: Structural difference between Meta-II and Opsin ${ }^{*}$ inferred from WAXS. Displaced helices III and V in Meta-II (yellow) are restored to dark structure in Opsin* (cyan), which is likely to destabilize the $\mathrm{G}$ protein activating conformation. 
TOC Graphic
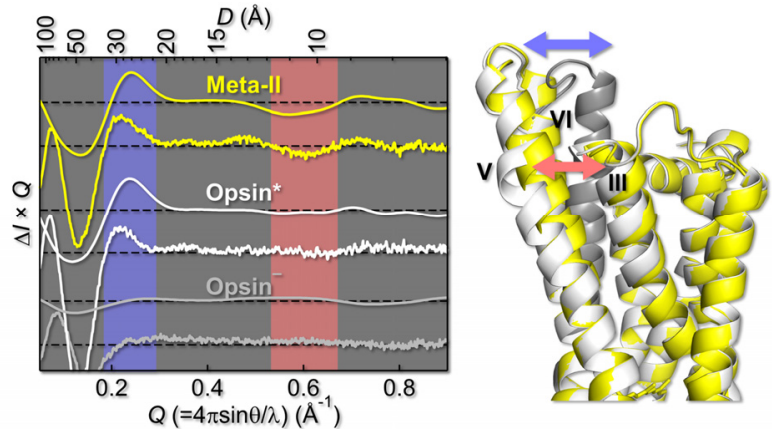


\section{Supporting Information}

\section{Conformational Differences among Metarhodopsin I, Metarhodopsin II, and Opsin Probed by Wide-Angle X-ray Scattering}

Yasushi Imamoto, ${ }^{*} \dagger$ Keiichi Kojima, ${ }^{\dagger}$ Toshihiko Oka, ${ }^{\ddagger} \S$ Ryo Maeda, ${ }^{\dagger}$ and Yoshinori Shichidall

†Department of Biophysics, Graduate School of Science, Kyoto University, Kyoto 606-8502, Japan

*Department of Physics, Faculty of Science and ${ }^{\S}$ Nanomaterials Research Division, Research Institute of Electronics, Shizuoka University, Shizuoka 422-8529, Japan

"Research Organization for Science and Technology, Ritsumeikan University, Kusatsu, Shiga 525-8577, Japan

Calculation of the Difference WAXS Curves. WAXS curves were calculated using CRYSOL software and the crystal structures listed in Table S1. The difference WAXS curves for the formation of photoproducts were calculated by subtracting the WAXS curve of the dark state from those of the photoproducts, followed by multiplication by $Q(\Delta I \times Q$ vs $Q)$ (Figures $2 \mathrm{~b}$ and $\mathrm{S} 3$ ).

Table S1: Calculation of difference WAXS curves using crystal structures.

\begin{tabular}{|c|c|c|c|}
\hline \multirow{2}{*}{ State } & \multicolumn{2}{|c|}{ PDB ID } & \multirow{2}{*}{$\begin{array}{l}\text { Amino acid residues and hetero atoms for } \\
\text { calculation }\end{array}$} \\
\hline & Photoproducts & Dark & \\
\hline Batho / Dark & $2 \mathrm{G} 87$ & $1 \mathrm{U} 19$ & \multirow{2}{*}{ Ac, Met1 - Ala348, Retinal (chain A) } \\
\hline Lumi / Dark & 2HPY & $1 \mathrm{U} 19$ & \\
\hline Meta- $\mathrm{II}_{\mathrm{a}} /$ Dark & $2 \mathrm{I} 37$ & $2 \mathrm{I} 36$ & $\begin{array}{l}\text { Ac, Met1 - Gly121, Ser127 - Val230, } \\
\text { Ala235, Thr242 - Cys322 (chain C) }\end{array}$ \\
\hline Meta-II / Dark & $3 \mathrm{PXO}$ & 1U19 & Met1 - Asn326, Retinal \\
\hline Opsin / Dark & 3CAP & 1U19 & Met1 - Asn326 (chain A) \\
\hline $\begin{array}{c}\text { Meta-II / Dark }{ }^{\mathrm{a}} \\
\text { (E113Q/N2C/D282C) }\end{array}$ & $2 \times 72$ & $1 \mathrm{GZM}$ & Met1 - Asn326, Retinal \\
\hline $\begin{array}{c}\text { Meta-II / Dark }^{\mathrm{a}} \\
(\mathrm{M} 257 \mathrm{Y} / \mathrm{N} 2 \mathrm{C} / \mathrm{D} 282 \mathrm{C})\end{array}$ & $4 \mathrm{~A} 4 \mathrm{M}$ & $1 \mathrm{GZM}$ & Met1 - Asn326, Retinal \\
\hline Meta-II / Dark ${ }^{\mathrm{a}}$ & $3 \mathrm{PXO}$ & $1 \mathrm{GZM}$ & Met1 - Asn326, Retinal \\
\hline
\end{tabular}

${ }^{\mathrm{a}}$ Figure S3 
Modeling of WAXS Curves of Meta-II. To identify the region responsible for the scattering intensity changes in difference WAXS curves between Meta-II and dark state, model structures were generated and difference WAXS curves were deduced by CRYSOL. From 1 U19 and 3PXO, the atoms for amino acid residues 1-326 and retinal were extracted, and overlapped using the "align" method of PyMol software (https://pymol.org) for all atoms. Then the region listed in Table S2 was interchanged between 1U19 and 3PXO, and difference WAXS curves were calculated (Figure 3).

Table S2: Calculation of difference WAXS curves using model structures.

\begin{tabular}{|c|c|c|}
\hline Replaced site & Abbreviation & Replaced amino acid residues \\
\hline Helix I & H1 & Glu33 - His65 \\
\hline Helix II & H2 & Thr70 - Gly101 \\
\hline Helix III & H3 & Phe105 - Cys140 \\
\hline Helix IV & H4 & Gly149 - Val173 \\
\hline 2nd Extracellular Loop & ECL2 & Gly174 - Pro194 \\
\hline Helix V & H5 & Glu201 - Gln236 \\
\hline Helix VI & H6 & Ala241 - His278 \\
\hline Helix VII & H7 & Gly284 - Met309 \\
\hline Helix VIII & H8 & Asn310 - Cys322 \\
\hline Intracellular half of Helix VI & H6IC & Ala241 - Ala260 \\
\hline Helix III and Helix V & H3\&H5 & $\begin{array}{l}\text { Phe105 - Cys140 } \\
\text { Glu201 - Gln236 }\end{array}$ \\
\hline Helix III and Extracellular part of & H3\&H5EC & $\begin{array}{l}\text { Phe105 - Cys140 } \\
\text { Glu201 - Gly224 }\end{array}$ \\
\hline Helix V & H3=H5 & $\begin{array}{l}\text { Val129 - Cys140 } \\
\text { Val218 - Thr229 }\end{array}$ \\
\hline Contact region of Helices III and V & &
\end{tabular}

Modeling of WAXS Curves of Lumi. To identify the region responsible for the scattering intensity changes in difference WAXS curves between Lumi and dark state, the region listed in Table S3 was interchanged between 1 U19 and 2HPY, and difference WAXS curves were calculated (Figure 4).

Table S3: Calculation of difference WAXS curves using model structures.

\begin{tabular}{|c|c|c|}
\hline Replaced site & Abbreviation & Replaced amino acid residues \\
\hline Helix I & H1 & Glu33 - His65 \\
\hline Helix II & H2 & Thr70 - Gly101 \\
\hline Helix III & H3 & Phe105 - Cys140 \\
\hline Helix IV & H4 & Gly149 - Val173 \\
\hline 2nd Extracellular Loop & ECL2 & Gly174 - Pro194 \\
\hline Helix V & H5 & His195 - Gln224 \\
\hline Helix VI & H6 & Ala241 - His278 \\
\hline Helix VII & H7 & Gly284 - Met309 \\
\hline Helix VIII & H8 & Asn310 - Cys322 \\
\hline & & Phe105 - Cys140 \\
Helices III, IV, V \& VI & H3-H6 & Gly149 - Val173 \\
& & His195 - Gln224 \\
\hline
\end{tabular}




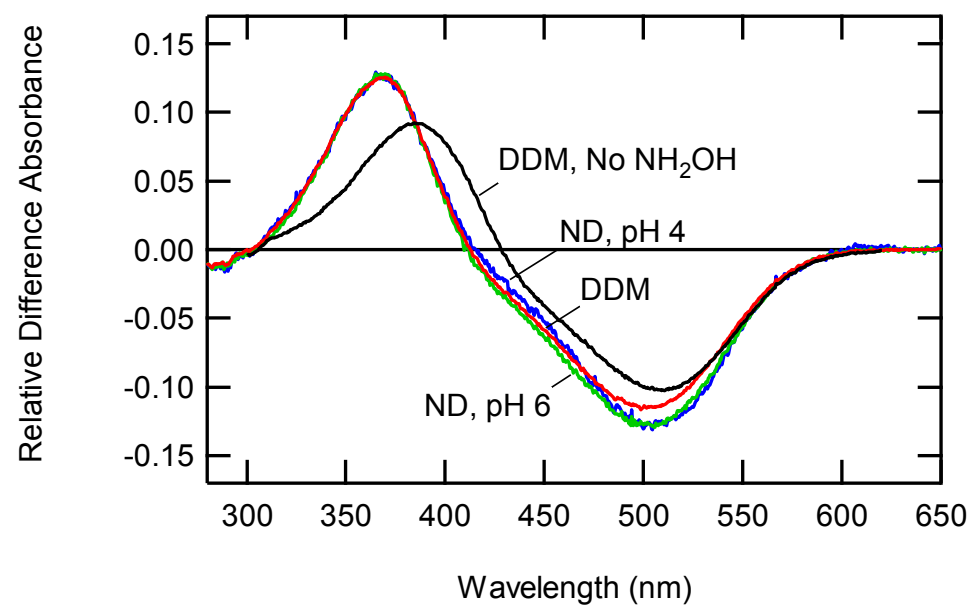

Figure S1: Formation of opsin in ND. Difference spectra before and 8 min after irradiation of Rh/ND were recorded in the presence of $100 \mathrm{mM}$ hydroxylamine at $\mathrm{pH} 4.0$ (blue) and $\mathrm{pH} 6.0$ (green). They were in good agreement with the difference spectrum between rhodopsin and all-trans-retinal oxime measured in DDM micelle (red). Difference spectrum between rhodopsin and Meta-II measured in the absence of hydroxylamine (black) is shown for comparison.

(a)

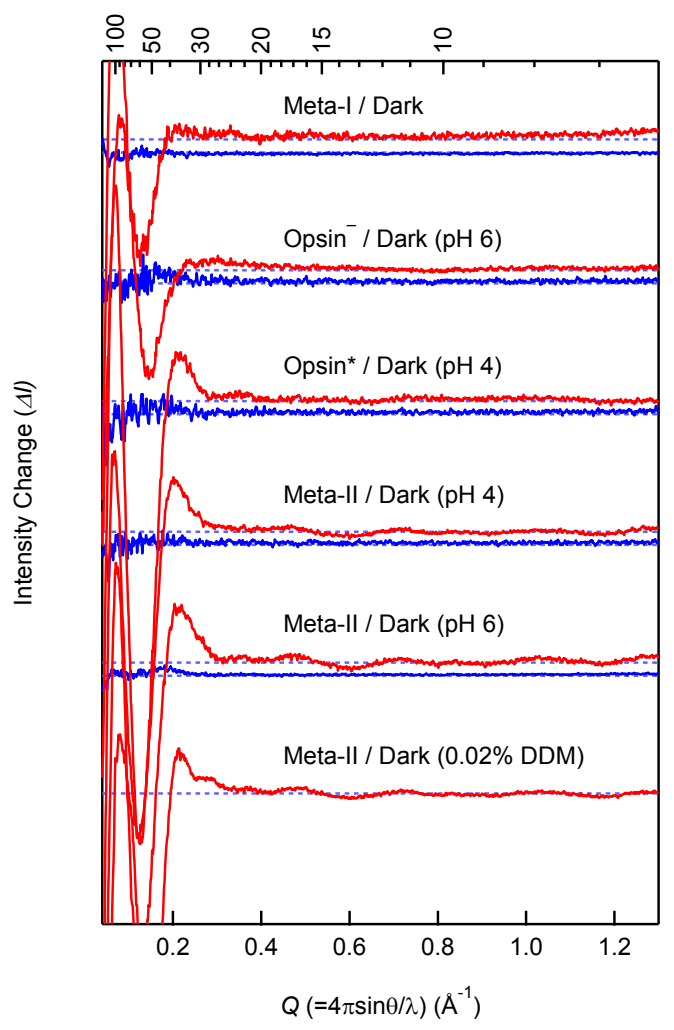

(b)

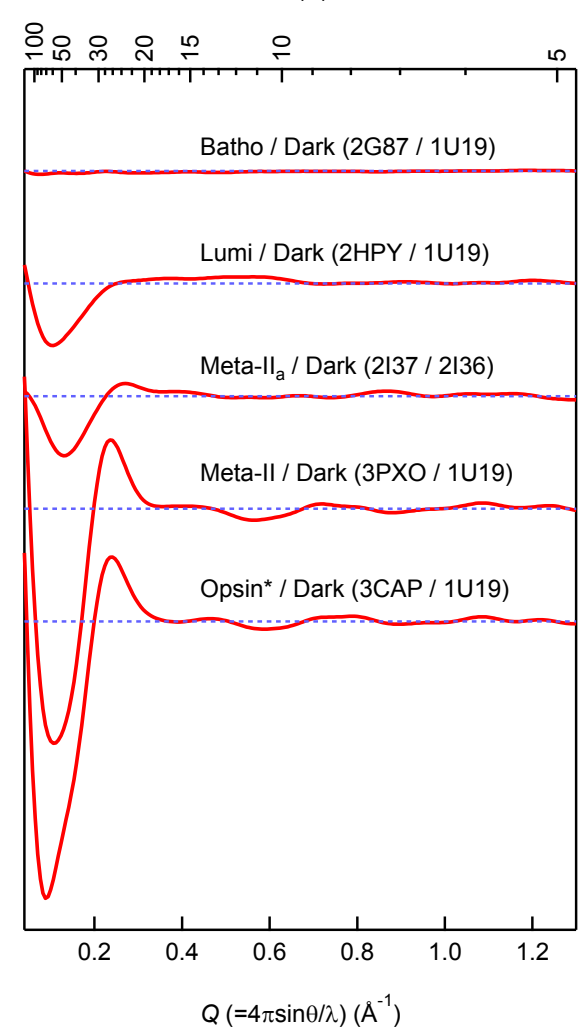

Figure S2: Difference WAXS curves shown in linear scale of Y-axis. 


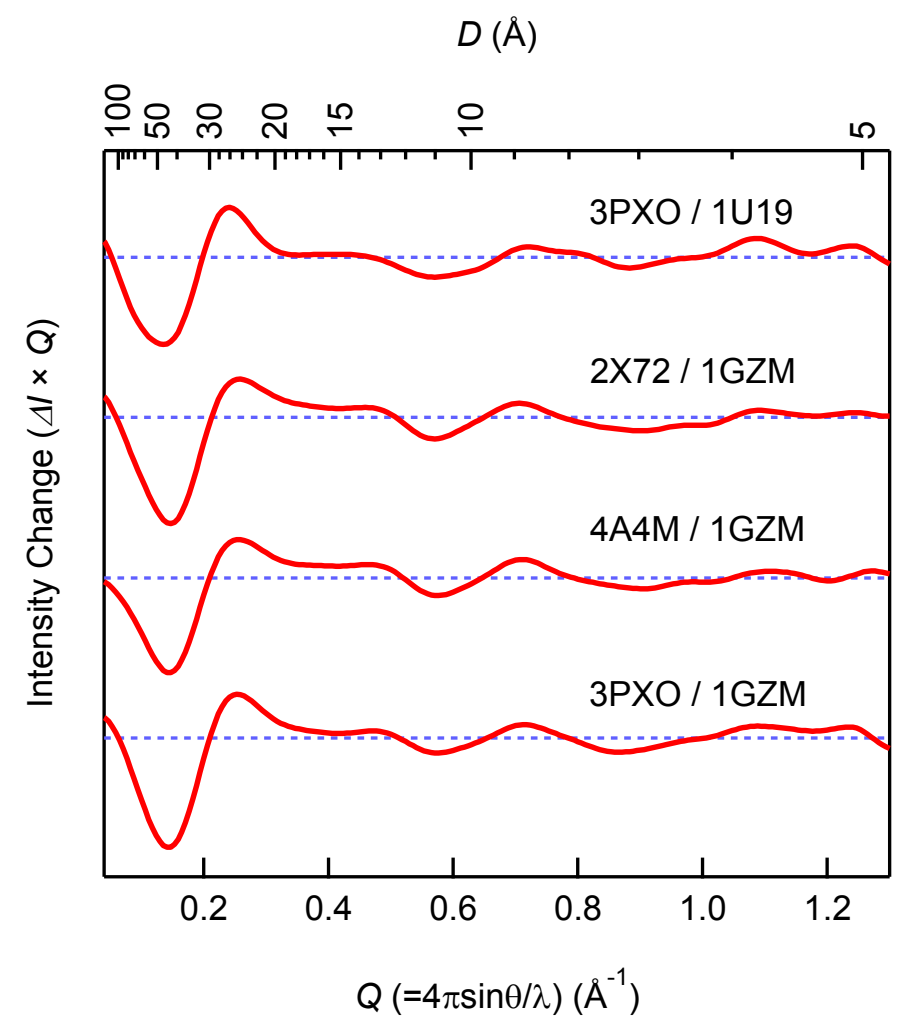

Figure S3: Difference WAXS curves for formtion Meta-II. Representative active structures (3PXO, Meta-II; 2X72, Meta-II from E113Q/N2C/D282C; 4A4M, M257Y/N2C/D282C) and dark-state structures (1U19 and 1GZM) were used for calculation. These curves are characterized by a $0.2 \AA^{-1}$ peak and a $0.6 \AA^{-1}$ valley. 